Cinémas

Revue d'études cinématographiques

Journal of Film Studies

\title{
Tinamer de Jean-Guy Noël : vers une nouvelle condition de réception
}

\section{Bernard Bérubé}

Volume 2, numéro 2-3, printemps 1992

Cinéma et Réception

URI : https://id.erudit.org/iderudit/1001085ar

DOI : https://doi.org/10.7202/1001085ar

Aller au sommaire du numéro

Éditeur(s)

Cinémas

ISSN

1181-6945 (imprimé)

1705-6500 (numérique)

Découvrir la revue

Citer cet article

Bérubé, B. (1992). Tinamer de Jean-Guy Noël : vers une nouvelle condition de réception. Cinémas, 2(2-3), 209-219. https://doi.org/10.7202/1001085ar

\section{Résumé de l'article}

En témoignant de la condition de son plaisir et de sa jouissance, la critique s'est prononcée sur la conformité du film Tinamer avec le cinéma dominant. Elle n'a pas vu le film comme une entité artistique ayant ses propres règles. L'atypisme de Tinamer ne demandait-il pas au spectateur de modifier son comportement, de produire un sens sans que l'esthétique du film ne soit soumise à l'idéologie du réalisme? L'analyse qui suit porte à conclure que l'atypisme de Tinamer conduit vers l'identification d'une nouvelle condition de réception que l'auteur tente de cerner. 


\title{
Tinamer de Jean-Guy Noël: vers une nouvelle condition de réception
}

\section{Bernard Bérubé}

\section{RÉSUMÉ}

En témoignant de la condition de son plaisir et de sa jouissance, la critique s'est prononcée sur la conformité du film Tinamer avec le cinéma dominant. Elle n'a pas vu le film comme une entité artistique ayant ses propres règles. L'atypisme de Tinamer ne demandait-il pas au spectateur de modifier son comportement, de produire un sens sans que l'esthétique du film ne soit soumise à l'idéologie du réalisme? L'analyse qui suit porte à conclure que l'atypisme de Tinamer conduit vers l'identification d'une nouvelle condition de réception que l'auteur tente de cerner.

\begin{abstract}
Taking note of the film Tinamer's focus on pleasure and enjoyment, critics have pronounced that it shows conformity to dominant cinema. But they have not considered this film as an artistic unity obeying to its own rules. Nor have they taken into account the fact that Tinamer's unusual nature requires a change in spectator behaviour: the film must be read in terms of an aesthetic other than the ideology of realism and verisimilitude. The analysis concludes that Tinamer's atypicality grounds the identification of a new condition of reception which the author undertakes to outline.
\end{abstract}


La journée des obsèques de sa mère, Tinamer adulte refuse de se rendre au salon mortuaire. Seule, dans son appartement, elle entreprend un voyage intérieur et renoue avec son enfance, époque où l'enfant $s$ 'abreuvait des mots et des images de son père. L'introspection de Tinamer ne rencontre pas les règles du cinéma dominant. Le récit ne part pas du point $\mathrm{A}$ pour arriver au point $\mathrm{Z}$. Le processus est circulaire: Tinamer part d'elle-même pour revenir à elle-même. Le réalisateur Jean-Guy Noël construit un film atypique ${ }^{1}$ qui ne donne pas à voir les souvenirs de la protagoniste, mais plutôt son inconscient. Pour illustrer cette démarche, le réalisateur a recours à des procédés inhabituels. Animation, conte, mime et danse «bricolent» un film qui fragmente la linéarité du récit.

Dans leur ensemble, les critiques cinématographiques furent défavorables à Tinamer. Ces quelques titres en témoignent: «Tinamer est-ce bien du cinéma?» (Le Matin) — «Tinamer: imaginaire et poésie sans magie» (Journal de Montréal) «Tinamer, un message à l'accent rugueux» (La Presse) «Vigneault gâche la sauce» (Le Soleil) - «Le poids des rêves» (Le Devoir). Ce que l'on reproche au film semble impliquer, en termes idéologiques, les choix esthétiques du cinéaste, le film modifiant les rapports film/spectateur. Une analyse du discours de la critique permet de mieux circonscrire ce point de vue. Il ne s'agit pas de reprocher à la critique d'avoir reçu le film comme elle l'a reçu, mais bien d'en comprendre les mécanismes et à quoi ils renvoient. Loin de nous l'idée de faire de Tinamer un chef d'œuvre que personne n'a su déchiffrer.

Tourné en 1986, le film sort sur nos écrans en 1988 pour seulement quatre semaines dans une seule salle à Montréal. La libre adaptation cinématographique du roman L'Amélanchier de Jacques Ferron est projetée sans tambour ni trompette, même si le coût de la réalisation est relativement élevé. On parle d'un budget de près de deux millions de dollars. Fait inusité, la couverture de presse du tournage est plus importante en terme de quantitatif que celle de la sortie du film (exception faite de la critique). Tinamer ne semble pas bénéficier du soutien normalement accordé à un film lors de sa sortie.

Le discours de la critique est étonnamment homogène. Les 
reproches formulés sont partout sensiblement les mêmes. L'article de Marcel Jean paru dans Le Devoir, «Le poids des rêves», regroupe les principaux arguments de ce discours. Afin de mieux en évaluer la portée, l'objet de notre analyse s'élaborera principalement à partir de cet article.

Marcel Jean écrit que «(...) le film de Jean-Guy Noël est décevant, même si le film tente d'aller à l'encontre de l'esthétique dominante». Il n'est pas le seul à reconnaître l'atypisme de Tinamer. Par exemple, dans 24 Images, Gilles Marsolais parle du cinéma singulier de Jean-Guy Noël. Cependant, une fois la différence admise, Marcel Jean et Gilles Marsolais se servent de critères relevant du cinéma dominant pour juger une œuvre qui s'en éloigne. Il y a donc contradiction entre ce qui paraît être une acceptation d'une nouvelle esthétique et ce qui dans les faits se révèle être un refus de celle-ci².

Un bon nombre de critiques, entre autres Bérubé, Jean, Perrault, Roberge, doutent même que Tinamer soit un film, soit du cinéma, que l'œuvre de Ferron ait été adaptable. Pierre Roberge de La Presse canadienne écrit: «Tinamer se laisse quand même regarder, il est plaisant d'entendre maximes et aphorismes rappelant qu'un bon roman est à l'origine du film. Mais est-ce bien du cinéma?» Pour sa part, Jean souligne que L'Amélanchier est un roman «sculpté de main de maître à même ce matériau si malléable qu'est la langue». Même si les critiques se défendent de comparer Tinamer au roman, ils valorisent l'œuvre littéraire au détriment du film. On présume des intentions de Noël. Par exemple, Gilles Marsolais dans 24 Images écrit: «C'est comme si le réalisateur s'était soudainement pris pour un autre, désireux de produire enfin "une grande œuvre" cinématographique à partir d'une œuvre littéraire préexistante fort estimable.»

Dans le discours de la critique, une des principales récurrences concerne le manque d'unité, l'éclatement du film. On souligne la difficulté de suivre le déroulement de l'histoire, de la reconstituer, de mettre bout à bout les différentes scènes. C'est en ces termes que Jean reproche à Tinamer son hétérogénéité:

(...) le premier problème est que Tinamer n'arrive jamais à prendre corps et à exister comme univers, comme lieu, comme film. Il lui manque cruellement l'homogénéité que procurait au roman la 
langue singulière de Ferron. Prises une par une, considérées en dehors de leur contexte, plusieurs scènes du film reposent sur des choix esthétiques défendables mais, mises à la suite des unes des autres, elles n'arrivent pas à tenir ensemble et à former ce monde de rêves auquel on aurait voulu nous faire croire.

Ce point de vue n'est pas sans rappeler celui de Jean Mitry ${ }^{3}$ pour qui un film devait être un monde en soi. Dans Essais sur la signification au cinéma Christian Metz, reprenant la théorie de Mitry, croit que «la théorie classique du cinéma (...) c'est la théorie du film-roman» (p. 12), du film qui se bâtit autour de «l'épopée romanesque». Les mots «film-roman» et «classique» renvoient à la valorisation de la forme parfaite à la Renaissance. L'emploi même de ces termes traduit une conception idéalisée du cinéma. En faisant référence au concept de classicisme, Metz semble signifier que le cinéma dont parle Mitry, c'est effectivement «le» cinéma. C'est de ce «lieu», de ce cinéma dont nous parle Jean.

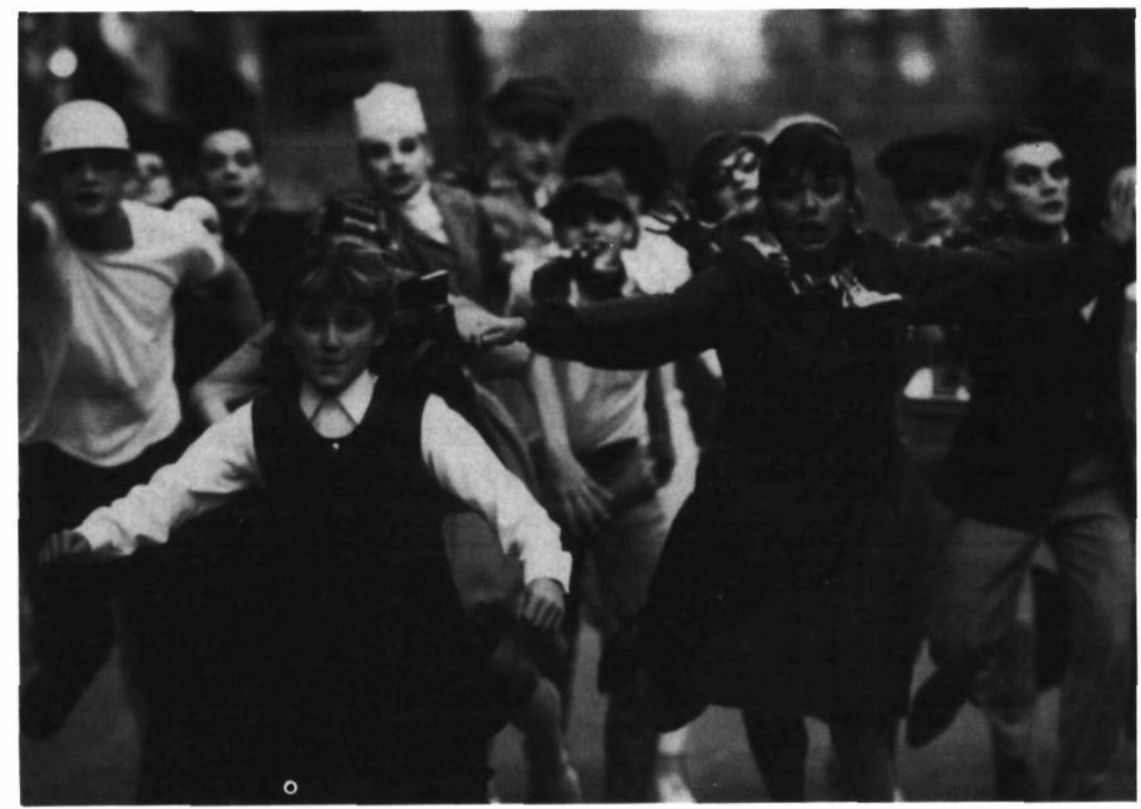

Une chorégraphie de Paul-André Fortier pour Tinamer Source: ACPAV, une photographie de Pierre Dury 
La critique déprécie les effets spéciaux, les images animées, car ceux-ci lui semblent «artificiels», «factices» comme du «travail de broderie», écrit Robert-Claude Bérubé dans Séquences. Jean abonde dans le même sens: «Éparpillé entre les séquences d'animation, les chorégraphies et les différents effets spéciaux, le récit que nous fait Noël colle mal à l'histoire racontée.» Ce que Jean nous dit en fait, c'est que ces éléments esthétiques ne sont pas assujettis à l'histoire. Il reproche aux effets de se donner en spectacle, de ne pas servir le code de la vraisemblance. Dans Tinamer, le mot Magia, qui se met à scintiller sur la pelouse sans que cela soit plausible, répond à un code différent. Le même effet dans un film du cinéma dominant aurait été justifié, par exemple, par le pouvoir magique d'un personnage comme c'est le cas dans E.T. Pierre Véronneau dans son analyse esthétique de l'atypisme de Tinamer explique ainsi le fonctionnement de la vraisemblance dans le cinéma dominant:

Par exemple une grande partie, la presque totalité de la sciencefiction repose sur cette dialectique: le spectateur sait qu'il est mis en présence d'une situation impossible mais l'imitation narrative la lui rend vraisemblable. Ce genre utilise les codes de la vraisemblance pour décrire l'imaginaire. C'est donc le langage qui détermine la perception et la lecture de l'œuvre; il établit la connexion entre lesystème spectatoriel et les propositions irréalistes du film (p. 155)

Le langage de Tinamer est trop éclaté pour permettre sa connexion au système spectatoriel en regard de la vraisemblance. Dans le film, l'animation matérialise des allégories. Celles-ci forment un collage hétéroclite, et, ainsi, elles fragmentent la linéarité du récit. Quand Jean écrit qu'il «est difficile d'adhérer à ces fragments d'univers, difficile de croire, ne serait-ce qu'un instant, en ce pays des rêves où les bécassines côtoient les centaures et où passent, la nuit, des êtres aux yeux lumineux», il désapprouve non seulement les choix esthétiques de Noël, mais aussi la non-adhésion du réalisateur à l'idéologie du réalisme à partir de laquelle s'élabore la relation spectateur-film.

D'ailleurs, l'ensemble des critiques fait état de cette difficulté d'adhésion. En fait, ils se butent au caractère hétéroclite du film, 
ce qui rend plus difficile la production de sens. Le récit de Tinamer rassemble bien plus des moments qu'il ne raconte une histoire. À la rigueur, certaines scènes sont interchangeables. La structure circulaire du film brise sa linéarité. Même si le voyage introspectif de Tinamer adulte s'apparente à un flash-back, il s'en éloigne, car les souvenirs de Tinamer sont davantage des ombres que des souvenances. Denise Martel du Journal de Québec recommande au spectateur de regarder Tinamer «comme on lit un poème». En fait, elle suggère au spectateur de changer son comportement.

Les ruptures abondent dans le film. Elles fragmentent la narration, le temps, l'espace, etc. D'ailleurs, Jean dénonce longuement une certaine rupture de l'espace filmique. Il reproche à Noël de ne pas avoir clairement situé la ville par rapport à la banlieue où habite Tinamer. Il affirme qu'il y a même confusion, que des plans se contredisent. C'est qu'il confronte deux plans, un premier que l'on suppose être le point de vue de Tinamer et un second plan tourné en focalisation zéro. Comment peut-on comparer deux points de vue différents? Comment savoir si la ville vue par Tinamer est réelle ou fictive, compte tenu du brouillage qu'excerce délibérément Noël entre l'espace réel et l'espace imaginaire? Noët ne nous fait pas revoir l'enfance de Tinamer, il nous donne à voir son inconscient. Il y a distorsion du point de vue. Chez Tinamer, le regard est introspectif. D'ailleurs, il contribue à structurer l'espace, entre autres, les rapports champ/ hors champ. Le hors champ, espace de l'inconscient, lorsque révélé, devient l'espace champ, espace du conscient. L'inconscient devenant conscient, Tinamer réconcilie les deux mondes de son enfance, le bon côté des choses et le mauvais. Ainsi, la manière dont est structuré l'espace relève de considérations autres que l'unité. Jean termine son argumentation en disant: «Un tel manque de rigueur sous des dehors anodins empêche le film de jouer sur les différences entre l'espace imaginaire de l'enfant et le véritable espace.» Mais de quelle rigueur s'agit-il? À quoi réfèret-elle, sinon au réalisme? Ainsi, le critique du Devoir endosse le point de vue de Christian Metz qui, dans Essais sur la signification au cinéma, déconseille l'emploi au cinéma des images imaginaires car «le cinéma est trop "réel" pour traduire efficacement les visées 
imaginaires de ses héros» (p. 43). Metz va même plus loin en affirmant que pour ne pas enfreindre la convention spectatorielle de la vraisemblance, il vaut mieux ne pas rappeler au spectateur que ce qu'il voit est de l'ordre de l'imaginaire.

Jean sert une leçon de cinéma à Noël en paraphrasant Lubitsch: «Si vous savez filmer des hommes, vous saurez filmer des rêves.» En affirmant cela, il endosse en quelque sorte les réticences de Metz à employer les images subjectives et imaginaires, à moins que celles-ci ne s'assemblent en segments narratifs semblables à ceux des images de la réalité:

(...) c'est la suite d'images tout entière qui est mise une fois pour toutes au compte du récit fabuleux; à l'intérieur de cet imaginaire premier, toutes les images redeviennent «objectives» (p. 46).

Lorsque Tinamer enfant lance une balle et que celle-ci fait le tour de la lune et retombe sur terre, nous passons d'une image de la réalité à une image animée sans aucune transition, comme si l'une était naturellement le prolongement de l'autre. En quelque sorte, Noël naturalise les images animées (monde de l'imaginaire) et il «artificialise» les images de la réalité (monde réel). La critique est agacée de ne pas toujours pouvoir clairement disposer des éléments pour bien démarquer le rêve de la réalité. Le manque de repères dérange.

Jean termine par une grande diatribe sur le choix et la direction des acteurs. «Et c'est là, au moment de choisir les interprètes que l'échec de Tinamer s'est véritablement décidé.» Il reproche à Vigneault de jouer Vigneault, à Sarah Jeanne Salvy de ne pas passer la rampe et à Louise Portal de faire contre-emploi. Il affirme que «s'ils [comédiens] avaient été mieux maîtrisés, ils auraient pu donner au film l'unité qui lui fait tant défaut». Il traite de la direction des acteurs, du traitement de la voix en fonction de l'harmonie du film et non en fonction de l'esthétique même de l'œuvre. Les critiques qui décrient le jeu des acteurs déprécient plus particulièrement le jeu de Vigneault. Ils lui reprochent de pas faire «vrai», de «conter», de «faire de la poésie.» ${ }^{4}$ Aux yeux de ces critiques, Vigneault ne fait pas réaliste, ne joue pas selon les standards habituels. Dans son analyse esthétique de Tinamer, Pierre Véronneau justifie le traitement de la voix en évoquant la distorsion 
du point de vue: «Vignault (sic) joue faux, Portal joue faux mais ils jouent vrai tous les deux. Car ils sont tous les deux exagérés dans la mémoire de Tinamer. Double distorsion qui rend le film encore plus déroutant» (p. 163).

Au cinéma dominant, ce sont les histoires qui engendrent les émotions de masse. Selon René Payant:

(...) le cinéma est rapidement et fermement devenu un objet de consommation de masse. Processus de massification, créant l'illusion d'une communatté des récepteurs pour lesquels le désir de réalisme iconique et narratif sert de ciment... L'organisation de l'hétérogénéité du système cinématographique s'est développée dans ce sens, construisant la synthèse harmonieuse de l'image, des voix et de la musique en vue de l'effet de réalisme (p. 596).

Quant au nouveau cinéma selon Pascal Bonitzer, il «casse les plans d'émotion, il invente de nouveaux rapports entre les plans, il décolle le cinéma des "émotions communes" pour produire des sensations nouvelles, moins identifiables, moins identificatoires» (p. 143). Le cas de Tinamer illustre bien ces enjeux.

En témoignant de la condition de son plaisir et de sa jouissance, la critique s'est prononcée sur le manque de conformité de Tinamer avec le cinéma dominant ${ }^{5}$. Elle n'a pas vu le film comme une entité artistique ayant des règles différentes et qui requiert du spectateur un changement de comportement. La réception de Tinamer cristallise les rapports entre culture de masse et pluralisme. Dans le cinéma dominant, les conventions de la réception sont en partie dictées par le réalisme. Le spectateur se prononce sur la conformité du film avec les normes. En fait, la culture de masse est contre le pluralisme, même si elle fait croire à la différence. Walter Benjamin constate:

Au cinéma le public ne sépare pas la critique de la jouissance. L'élément décisif est ici que, plus que nulle part ailleurs, les réactions individuelles, dont l'ensemble constitue la réaction massive du public, y sont déterminées, dès le point de départ, par l'immédiate virtualité de leur caractère collectif (p.114).

Le cinéma atypique comme dans le cas de Tinamer ne se limite pas à être un nouveau style. Il remet en question non seulement les éléments esthétiques du cinéma dominant, mais l'idéologie du réalisme. Linéarité, homogénéité, histoire, narration, espace... 
sont brisés dans une perspective de renversement de l'ordre des choses. Ce nouveau cinéma, que l'on qualifie parfois de postmoderne, modifie les rapports entre le cinéma et le spectateur. Ainsi, nous pensons qu'il est plus approprié de parler de condition que de style. René Payant abonde dans ce sens:

(...) n'est pas non plus une affaire de style (...) Il concerne plutôt les systèmes interprétatifs et évaluatifs, c'est à dire la réception. La condition postmoderne c'est un devoir-inventer un nouvel ordre des choses et une nouvelle conception de l'ordre qui ne craint pas de remplacer l'idée de cohérence sans failles par celle de complexité (p. 599).

Tinamer rélève de ce nouveau cinéma qui, au-delà des différences esthétiques, laisse entrevoir des conditions différentes de création et de réception. Jean-Guy Noël a convié le spectateur à un film différent. L'œuvre s'ouvre ${ }^{6}$ et interpelle le spectateur non pas seulement en tant qu'individu-jouisseur, mais aussi en tant qu'individu-auteur. Bien que la contribution du spectateur ne soit pas concrète (le spectateur ne peut pas refaire le montage), Noël requiert de lui une collaboration théorique et mentale pour faire Tinamer. En fait, la condition de plaisir du spectateur, condition en partie créée par les émotions de masse et par la virtualité de leur caractère collectif, s'accompagne d'une condition qui requiert du spectateur une plus grande participation. Dans une certaine mesure, Tinamer témoigne d'un éclatement des schèmes dominants au profit de l'apparition d'une nouvelle condition de réception telle que l'a décrite le musicologue Henri Pousseur:

Les phénomènes n'étant plus enchaînés les uns aux autres par un déterminisme de terme à terme, c'est à l'auditeur de se placer volontairement au milieu d'un réseau de relations inépuisables, de choisir pour ainsi dire lui-même ses dimensions d'approche, ses points de repère, son échelle de référence, de tendre à utiliser simultanément le plus grand nombre d'échelles et de dimensions possibles, de dynamiser, de multiplier, d'écarquiller à l'extrême ses instruments de saisie (p. 15).

Après avoir été marquée par le positivisme et le cartésianisme, la culture est davantage influencée par la répartition statistique, l'indétermination, la méthodologie quantique et la contradiction. C'est en termes de possibilités que la culture se conçoit et perçoit 
le monde. La technologie numérique, l'interactivité et l'hypermédiatique répondent à cette multiplication des possibilités. Avec celles-ci le spectateur sera appelé à devenir un utilisateur. Les conventions qui régissent la réception seront appelées à se modifier. Alors, les configurations et les formations de sens, auxquelles nous sommes habituées, basculeront. Ce n'est pas uniquement l'influence des nouvelles technologies sur le cinéma qui expliquent les changements à venir, mais le fait qu'elles et lui appartiennent à la même civilisation. L'éclatement de Tinamer relèverait de ce fait de culture.

\section{Université de Montréal}

\section{NOTES}

1 Dans l'article intitulé «Jean-Guy Noël: la marginalisation d'un cinéma atypique» paru dans Le Cinéma québécois des années 80 (Montréal: Cinémathèque québécoise et Musée du cinéma, 1989), Pierre Véronneau analyse l'esthétique de Tinamer en regard de l'atypisme.

2 Cette façon de faire rappelle la démarche de Heinrich Wolfflin, historien d'art (Renaissance et baroque, Paris: Poche, 1961). Ce dernier définit l'art baroque avec des données morphologiques de l'art renaissant. Il se défend bien de porter un jugement de valeur, attendu qu'il se sert de critères objectifs. Cependant, sa méthode le conduit à conclure que les œuvres baroques renient les acquis des œuvres renaissantes. Comment peut-il en être autrement?

3 Voir à ce sujet Jean Mitry, Esthétiqueetpsychologie ducinéma (Paris: Éditions universitaires, tome 1- 1963, tome 2 - 1965).

4 Le statut de Vigneault pose un problème d'identité aux yeux de certains critiques. Le texte le plus explicite à ce sujet est celui de François Bilodeau, «Comment dorer sa propre cage», Spirale (avril 1988).

5 Tout film qu'il soit commercial ou atypique n'est pas à l'épreuve de la critique. Tinamer n'est pas à l'abri du propre déséquilibre de son système et donc de la critique défavorable. Encore faudrait-il établir des critères selon le système qui est propre au film.

6 Cette ouverture s'apparente à celle des œuvres qui s'offrent à une pluralité d'organisations et qu'Umberto Eco qualifie «d'œuvre ouverte» à la commu- 
nication (L'Oeuvre ouverte, Paris: Seuil, 1965).

\section{OUVRAGES CITÉS}

Benjamin, Walter. «L'Oeuvre d'art à l'ère de la reproductibilité technique», Essais 2 - 1935-1940. Paris: Denoë1/Gonthier, 1983.

Bérubé, Robert-Claude. «Tinamer ». Séquences, n 134 (juin 1988).

Bonitzer, Pascal. Le Champ aveugle. Paris: Cahiers du cinéma/Gallimard, 1982.

Gaudreault, Léonce. «Vigneault gâche la sauce». Le Soleil (5 mars 1988).

Jean, Marcel. «Le Poids des rêves». Le Devoir (30 janvier 1988).

Marsolais, Gilles. «Tinamer ». 24 Images, $\mathrm{n}^{\circ} 36$ (nov. 87-jan. 88).

Martel, Denise. «L'Amélanchier. Le réalisateur inspiré par l'œuvre de Ferron». Journal de Québec (27 février 1988).

Metz, Christian. Essaissurlasignificationaucinéma, tome 2. Paris: Klincksieck, 1972.

Payant, René. Vedute, Pièces détachées sur l'art. Montréal: Trois, 1987.

Perrault, Luc. «Tinamer. Un message àl'accent rugueux».La Presse (30janvier 1988).

Pousseur, Henri. «Vers un nouvel univers sonore». Esprit (janvier 1960).

Roberge, Pierre. «Tinamer: est-ce bien du cinéma?» (Presse canadienne). Le Matin (Moncton, 21 janvier 1988).

Véronneau, Pierre. «Jean-Guy Noël: la marginalisation d'un cinéma atypique», Le Cinéma québécoisdes années 80 . Montréal: Cinémathèque québécoise et Musée du cinéma, 1989.

Villeneuve, Paul. «Tinamer : imaginaire et poésie sans magie». Journal de Montréal (30 janvier 1988). 